\title{
EDITORIAL
}

\section{Unveiling ayahuasca psychopharmacology: the accomplishments of Jordi Riba (1968-2020)}

\author{
Rafael G. dos Santos, ${ }^{1,2}$ (ID Draulio B. Araujo, ${ }^{3}$ (ID Rafael F. Sanches, ${ }^{1,2}$ (ID Jaime E. Hallak ${ }^{1,2}$ (ID \\ ${ }^{1}$ Departamento de Neurociências e Ciências do Comportamento, Faculdade de Medicina de Ribeirão Preto, Universidade de São Paulo, \\ Ribeirão Preto, SP, Brazil. ${ }^{2}$ Instituto Nacional de Ciência e Tecnologia Translacional em Medicina (INCT-TM), Ribeirão Preto, SP, Brasil. \\ ${ }^{3}$ Instituto do Cérebro, Universidade Federal do Rio Grande do Norte, Natal, RN, Brazil.
}

On August 14, 2020, Jordi Riba (Barcelona, b. 1968) passed away, which is a great loss to his family, friends, and colleagues worldwide.

Professor Riba was a researcher in the Department of Neuropsychology and Psychopharmacology, Maastricht University (Maastricht, Netherlands), and former associate professor in the Department of Pharmacology, Therapeutics and Toxicology, Universitat Autònoma de Barcelona (UAB)/Hospital de la Santa Creu i Sant Pau (HSCSP). He was a Catalan and the first researcher to conduct placebo-controlled trials with ayahuasca in healthy volunteers, ${ }^{1}$ leading the first trials on the safety and tolerability, pharmacokinetics, and subjective and neurophysiological effects of ayahuasca. His studies on ayahuasca began with his Ph.D. dissertation entitled "Human pharmacology of ayahuasca," concluded in 2003 under the supervision of Professor Manuel José Barbanoj Rodriguez (MD, UAB/HSCSP). ${ }^{2}$ At the time of his death, Professor Riba was a researcher at Maastricht University, where he worked in the field of human psychedelic/hallucinogen research.

His seminal work suggested for the first time that freezedried ayahuasca could be safely administered to healthy humans in a controlled context and that the inhibiting properties of monoamine oxidase in ayahuasca allow DMT to reach the brain. He also conducted the first neuroimaging study with ayahuasca, showing that it increases brain activity in the frontal and paralimbic areas. ${ }^{3}$ These studies included ayahuasca in the list of substances of interest to the field of psychedelic sciences, which includes LSD and psilocybin. Prof. Riba made a significant contribution to the science of human interaction with other substances, such as cocaine, Salvia divinorum, 5-MeO-DMT, and cannabinoids.

Furthermore, Dr. Riba's studies opened the doors for other researchers in the field, especially in Brazil, which he visited on a number of occasions for scientific collaboration. Between 2006 and 2012, he was the Ph.D. supervisor of Rafael Santos at the Universitat Autònoma

Correspondence: Rafael Guimarães dos Santos, Departamento de Neurociências e Ciências do Comportamento, Faculdade de Medicina de Ribeirão Preto, Universidade de São Paulo, Hospital das Clínicas, Terceiro Andar, Av. Bandeirantes, 3900, Ribeirão Preto, SP, Brazil.

E-mail: banisteria@gmail.com

Submitted Aug 17 2020, accepted Aug 17 2020, Epub Oct 072020. de Barcelona. The trials from this thesis showed for the first time that two consecutive doses of ayahuasca do not produce tolerance, and that ayahuasca modulates the HPA-immune axis. ${ }^{4}$ Professor Riba was also a close collaborator in the first trials of ayahuasca in the treatmentresistant major depression, which lead to the publication of the first randomized controlled trial with a psychedelic substance for a major psychiatric condition. ${ }^{5}$ These trials not only showed that a single dose of ayahuasca was safe for use in patients with major depression, but that it also induced fast-acting (hours) and enduring (days/weeks) antidepressive effects. He also collaborated with the Instituto Nacional de Ciência e Tecnologia Translacional em Medicina (INCT-TM) and was the postdoctoral supervisor of Rafael Sanches in 2013. He will certainly remain present in our group through these studies, as well as ideas.

In 2017, Rolling Stone included Dr. Riba among the 25 most influential persons in science (https://bit.ly/rollsto nesjriba). Dr. Riba had a peculiar sense of humor and was a rigorous scientist. Although he brought ayahuasca to the laboratory, he always treated it with the greatest respect. As in our studies here in Brazil, the ayahuasca he used was obtained from Brazilian ayahuasca religious practitioners. He was very enthusiastic about this field of research, and his approach to ayahuasca was to improve our current society, always respecting ayahuasca culture. He was deeply interested in indigenous cultures, which probably initiated his interest in ayahuasca.

His soul was enamored by challenges, achievements, and the search for new frontiers. At sea, he was a professional scuba diver and an experienced sailor. In the sky, he survived a paraglider accident and piloted airplanes with all the rigor of scientific research. As a child he heard stories of his father's expeditions in Africa, and as he grew he got a taste for it and travelled the world. In Brazil for example, he slept in hammocks in the Amazon jungle, saw Iguaçu Falls, and floated down rivers in the Brazilian Pantanal.

How to cite this article: dos Santos RG, Araujo DB, Sanches RF, Hallak JE. Unveiling ayahuasca psychopharmacology: the accomplishments of Jordi Riba (1968-2020). Braz J Psychiatry. 2021; 43:565-566. http://dx.doi.org/10.1590/1516-4446-2020-0021 
He was very enthusiastic about science in general, loved to eat rovellons (a typical Catalonian mushroom) with Spanish wine. Deeply knowledgeable, he enjoyed history and arts. He will be missed by all of us but will still be in our hearts and minds. Moltes gràcies Jordi.

\section{Disclosure}

The authors report no conflicts of interest.

\section{References}

1 Riba J, Rodríguez-Fornells A, Urbano G, Morte A, Antonijoan R, Montero $M$, et al. Subjective effects and tolerability of the South
American psychoactive beverage ayahuasca in healthy volunteers. Psychopharmacology (Berl). 2001;154:85-95.

2 Riba J. Human pharmacology of ayahuasca [dissertation]. Barcelona: Universitat Autònoma de Barcelona; 2003.

3 Riba J, Romero S, Grasa E, Mena E, Carrió I, Barbanoj MJ. Increased frontal and paralimbic activation following ayahuasca, the pan-Amazonian inebriant. Psychopharmacology (Berl). 2006;186: 93-8.

4 Dos Santos RG, Grasa E, Valle M, Ballester MR, Bouso JC, Nomdedéu JF, et al. Pharmacology of ayahuasca administered in two repeated doses. Psychopharmacology (Berl). 2012;219: 1039-53.

5 Palhano-Fontes F, Barreto D, Onias H, Andrade KC, Novaes MM, Pessoa JA, et al. Rapid antidepressant effects of the psychedelic ayahuasca in treatment-resistant depression: a randomized placebocontrolled trial. Psychol Med. 2019;49:655-63. 